\title{
Expresión tumoral de BRCA1 y resultados clínicos en pacientes uruguayas diagnosticadas de cáncer de mama antes de los 40 años
}

\author{
Silvina Malvasio*, Natalia Camejo ${ }^{\dagger}$, Carina Di Matteo ${ }^{\ddagger}$, Benedicta Caserta§, \\ Nora Artagaveytia", Cecilia Castillo** , Andrea Schiavone ${ }^{\dagger \dagger}$, Roberto Notejane ${ }^{\ddagger \neq}$,

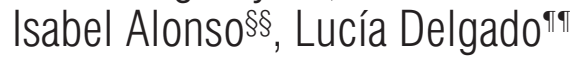

\section{Resumen}

Las mutaciones de BRCA1 son raras en el cáncer de mama (CM) esporádico; sin embargo, su expresión a nivel tumoral se encuentra disminuida o ausente en $30 \%-50 \%$ de los casos.

Objetivo: valorar la expresión tumoral de BRCA1 por inmunohistoquímica (IHQ) en mujeres uruguayas diagnosticadas de CM antes de los 40 años.

Material y método: se incluyeron pacientes diagnosticadas de CM antes de los 40 años. Se utilizaron los anticuerpos monoclonales anti-BRCA1 MS110 contra el extremo N-terminal y GLK-2 contra el extremo C-terminal. Se calculó la sobrevida global (SVG) y la sobrevida libre de enfermedad (SVLE), para la construcción de las curvas se utilizó el método de Kaplan-Meier y la diferencia de sobrevida se evaluó mediante el test de log rank.

Resultados: se incluyeron 40 pacientes, la SVG y la SVLE a cinco años fueron de $73 \%$ y $60 \%$ respectivamente. La expresión de BRCA1 mediante GLK-2 fue $\leq 10 \%$ en 16 de las 40 pacientes (40\%). La SVG y la SVLE a cinco años para las pacientes con expresión $\leq 10 \%$ fue de $56 \%$ vs $85 \%$ para las pacientes con expresión $>10 \%$ ( $p=0,015)$ y de $40 \%$ vs $72 \%(p=0,034)$ respectivamente. La expresión de BRCA1 mediante MS110 fue $\leq 10 \%$ en 11 de las 40 pacientes (27,5\%). No se encontraron diferencias en la SVG ni en la SVLE a cinco años con este marcador.

Conclusión: la pérdida de la expresión tumoral de BRCA1 determinada mediante GLK-2 se encontró en el 40\% de las pacientes incluidas y se asoció a una menor SVG y SVLE, por lo que podría tener un valor pronóstico desfavorable en estas pacientes.

\footnotetext{
* Asistente del Servicio de Oncología Clínica, Hospital de Clínicas, Facultad de Medicina, Universidad de la República. Uruguay.

† Prof. Adjunta del Servicio de Oncología Clínica, Hospital de Clínicas, Facultad de Medicina, Universidad de la República. Uruguay.

‡ Prof. Agregada del Departamento de Anatomía Patológica, Hospital de Clínicas, Facultad de Medicina, Universidad de la República. Uruguay.

$\S$ Prof. Adjunta del Departamento de Anatomía Patológica, Hospital de Clínicas, Facultad de Medicina, Universidad de la República. Uruguay.

Jefe del Dpto. de Citología y Anatomía Patológica del Hospital de la Mujer, Centro Hospitalario Pereira Rossell. Uruguay.

II Prof. Adjunta del Departamento Básico de Medicina, Hospital de Clínicas, Facultad de Medicina, Universidad de la República. Uruguay.

** Prof. Adjunta del Servicio de Oncología Clínica, Hospital de Clínicas, Facultad de Medicina, Universidad de la República. Uruguay.

†† Ex Asistente del Servicio de Oncología Clínica, Hospital de Clínicas, Facultad de Medicina, Universidad de la República. Uruguay.

¥¥ Oncólogo Médico. Jefe del Servicio de Oncología Clínica del Centro de Asistencia Médica del Sindicato Médico del Uruguay (CASMU-IAMPP). Uruguay.

$\S \S$ Oncólogo Médico. Jefe del Servicio de Oncología Clínica del Hospital de la Mujer, Centro Hospitalario Pereira Rossell. Uruguay.

ๆศा Prof. Directora del Servicio de Oncología Clínica, Hospital de Clínicas, Facultad de Medicina, Universidad de la República. Uruguay.

Servicios responsables: Servicio de Oncología Clínica, Hospital de Clínicas "Dr. Manuel Quintela". Universidad de la República. Hospital de la

Mujer, Centro Hospitalario Pereira Rossell. CASMU-IAMPP. Montevideo, Uruguay.

Correspondencia: Dra. Silvina Malvasio. Servicio de Oncología Clínica. Hospital de Clínicas, Avda. Italia s/n. CP 11600. Montevideo, Uruguay.

Correo electrónico: silvivasio@gmail.com

Los autores declaran no tener conflicto de intereses.

Este trabajo fue aprobado por los comités de ética de todas las instituciones participantes.

Recibido: $14 / 8 / 19$

Aprobado: 23/10/19
} 
Palabras clave: Neoplasias de la mama Genes BRCA1

Inmunohistoquímica

Mujeres jóvenes

$\begin{array}{ll}\text { Key words: } & \text { Breast neoplasms } \\ & \text { BRCA1 genes } \\ & \text { Immunohistochemistry } \\ & \text { Young women }\end{array}$

\section{Introducción}

En nuestro país el cáncer de mama (CM) es la principal causa de muerte por cáncer en la mujer y con exclusión del cáncer de piel no melanoma es el cáncer más frecuente en el sexo femenino, dando cuenta de aproximadamente 650 muertes por año y 1.800 casos nuevos al año, de los cuales 99 corresponden a mujeres menores de 40 años (período 2007-2011), lo que representa aproximadamente $5,4 \%$ de todos los casos diagnostica$\operatorname{dos}^{(1,2)}$.

Aunque el CM no es habitual en mujeres menores de 40 años, involucra a mujeres en edad fértil que se encuentran en plena actividad laboral, por lo que su diagnóstico tiene un mayor impacto a nivel personal, familiar y social.

La edad es un factor pronóstico desfavorable para estas pacientes, siendo las tasas de recaída local y a distancia y mortalidad mayor en las mujeres jóvenes ${ }^{(3-9)}$. Así, en la práctica clínica habitual la edad de la paciente es un factor de peso mayor y frecuentemente las pacientes jóvenes reciben tratamientos sistémicos más agresi$\operatorname{vos}^{(10)}$

Previamente nuestro grupo reportó las características clínico patológicas; el estatus de HER2, receptores de estrógenos (RE) y receptores de progesterona (RP) estudiados mediante inmunohistoquímica (IHQ); la sobrevida libre de enfermedad (SVLE) y la sobrevida global (SVG) en pacientes uruguayas diagnosticadas de $\mathrm{CM}$ antes de los 40 años ${ }^{(11)}$. La SVG y la SVLE a cinco años fueron $79 \%$ y $72 \%$, respectivamente, menor a la reportada en pacientes de la población general y de mayor rango etario.

Si bien la peor evolución se ha vinculado al retraso en el diagnóstico, la mayoría de nuestras pacientes fueron diagnosticadas en estadios localizados y la totalidad realizó los tratamientos según las pautas vigentes. Por otra parte, la proporción de subtipos desfavorables (triple negativos [TN] y HER2+) fue mayor a lo reportado para mujeres de todas las edades con $\mathrm{CM}$, por lo que su peor evolución podría ser explicada por su perfil biológico.

En relación al riesgo genético, a pesar de que la mayor parte de los CM diagnosticados en pacientes jóvenes son esporádicos, estas pacientes tienen un mayor riesgo de presentar una predisposición hereditaria para el desarrollo de la enfermedad, siendo los genes BRCA1 y 2 (BReast CAncer 1 y 2) los que usualmente se encuentran implicados. Entre 11,6\% y $17 \%$ de las mujeres con diagnóstico de $\mathrm{CM}$ menores de 40 años presenta una mutación en algunos de estos genes $^{(12,13)}$. En nuestro estudio, el $17 \%$ de las pacientes incluidas presentaron AF significativos, lo cual, sumado a la temprana edad de aparición de cáncer en estas pacientes, sugeriría una predisposición hereditaria al CM o de ovario, o ambos.

BRCA1 es un gen supresor de tumor que cuando se encuentra mutado predispone al $\mathrm{CM}$ y otros tumores, en particular cáncer de ovario ${ }^{(14-17)}$.

Las mutaciones de línea germinal en estos genes explican aproximadamente el 20\%-40\% de los CM familiares. Además, su expresión se encuentra disminuida, sin mutación génica que lo explique, en aproximadamente $30 \%-50 \%$ de los $\mathrm{CM}$ esporádicos ${ }^{(18,19)}$.

El gen BRCA1 codifica una proteína nuclear que interactúa con un gran número de proteínas reguladoras que participan en procesos celulares fundamentales, tales como la reparación del ADN, la transcripción, el control del ciclo celular y la apoptosis. En particular, se ha demostrado que está implicada en la reparación del daño genético contribuyendo al mantenimiento de la integridad del genoma ${ }^{(20,21)}$.

Desde el punto de vista molecular no existe una clara caracterización de los tumores en las mujeres jóvenes. Si bien en la población general, 30\%-35\% de los CM esporádicos presentan pérdida de función de BRCA1, asociada a cambios epigenéticos, no hemos encontrado estudios en la literatura que reporten el nivel de expresión de BRCA1 en mujeres menores de 40 años con CM esporádico $^{(12,13,19,18,22)}$.

En el presente estudio se realiza la determinación de la expresión tumoral de BRCA1 mediante IHQ en las muestras de tumores de pacientes diagnosticadas de $\mathrm{CM}$ antes de los 40 años con el fin de analizar la correlación entre su grado de expresión y la evolución, siendo nuestro objetivo investigar si es un factor pronóstico de sobrevida en estas mujeres.

\section{Objetivo}

Evaluar el nivel de expresión de la proteína BRCA1 mediante IHQ e investigar si la expresión tumoral de BRCA1 es un factor pronóstico de la SVLE y la SVG a cinco años. 


\section{Material y método}

Se trata de un estudio retrospectivo en el cual se seleccionaron pacientes diagnosticadas de $\mathrm{CM}$ antes de los 40 años en el período comprendido entre el $1^{\circ}$ de enero de 2006 y el 31 de diciembre de 2012, con disponibilidad de muestra tumoral parafinada en archivo para realizar técnicas IHQ y que hubiesen dado su consentimiento informado. En caso de que la paciente hubiese fallecido, se solicitó consentimiento para la utilización de material de archivo (bloques de parafina) al familiar más cercano accesible. Las pacientes fueron asistidas en el Servicio de Oncología del Hospital Universitario de Clínicas, en el Hospital de la Mujer Paulina Luisi del Centro Hospitalario Pereira Rossell (CHPR) y en el Centro de Asistencia del Sindicato Médico del Uruguay (CASMU-IAMPP).

La recolección de datos se realizó a través del estudio minucioso de las historias clínicas.

Se obtuvieron los siguientes datos: edad de la paciente al diagnóstico; antecedentes gineco-obstétricos (edad de la menarca, estado menopáusico al diagnóstico, utilización de anticonceptivos orales, gestas, lactancia y duración de la misma); AF significativos (contando a la paciente, tres o más casos de $\mathrm{CM} /$ cáncer de ovario, al menos uno antes de los 50 años); tipo y grado histológico; tamaño tumoral; estado de los ganglios linfáticos axilares; estadio de acuerdo con la clasificación TNM; estatus de HER2, RE y RP estudiados mediante IHQ; tratamientos realizados (cirugía, tratamiento radiante o sistémico); recaída tumoral (local o sistémica).

Los estudios de IHQ para determinación del estatus de HER2, RE, RP y Ki 67 son parte del trabajo rutinario de los departamentos de anatomía patológica de las instituciones donde se asistieron las pacientes. El nivel de expresión de HER2 se determinó mediante Herceptest ${ }^{\mathbb{B}}$ (Dako, Carpinteria, CA, USA). Los informes de los resultados de la inmunomarcación para HER2 se basaron en las recomendaciones vigentes en el período en que fueron estudiadas las pacientes, considerándose positivo el resultado con inmunotinción 3+. En casos IHQ 2+ se definió el estatus HER 2 mediante Fluorescent In Situ Hybridization (FISH).

Se definieron tres subtipos tumorales en base a la expresión positiva o negativa por IHQ y FISH, en caso de ser necesario, de HER2, RE y RP:

1) RRHH +, HER2-: RE+ y RP+, HER2-; RE-/RP+, HER2-; RE+/RP-,HER2-.

2) HER2 positivo: HER2 positivo, RE/RP + o -.

3) RE-, RP-, HER2- (triple negativo).

Se calculó SVG y SVLE para la población total y según la expresión de BRCA1 por IHQ, utilizando el mé- todo de Kaplan-Meier y el test de log rank. Se usó un nivel de significancia estadística de $\mathrm{p}<0,05$. El paquete estadístico usado fue el SPSS 22.

La puesta a punto de la técnica y la determinación de BRCA1 mediante IHQ se realizó de forma centralizada, automatizado en equipo Autostainer Link 48. Se utilizaron los anticuerpos monoclonales anti-BRCA1 MS110 dirigido contra la región comprendida entre los 304 aminoácidos del extremo N-terminal y GLK-2 contra la región comprendida entre los aminoácidos 1839-1863, del extremo C-terminal. Para utilizar el anticuerpo monoclonal MS110 se empleó una dilución 1/100 y para el anticuerpo monoclonal GLK-2 se utilizó una dilución 1/50. Para analizar el nivel de expresión de la proteína BRCA1 se utilizaron los patrones acordados en la literatura considerando un resultado positivo cuando la inmunoexpresión nuclear o citoplasmática, o ambas, era $>10 \%$ y negativo cuando la expresión era $\leq 10 \%$ en el núcleo y citoplasma de las células tumorales ${ }^{(23-27)}$.

\section{Aspectos éticos}

El presente estudio se realizó de acuerdo a las normas éticas internacionales para las investigaciones biomédicas: Normas del MERCOSUR sobre regulación de los estudios clínicos y la Declaración de Helsinski de la Asociación Médica Mundial (atendiendo a su última enmienda de octubre del 2013). Se mantuvo el anonimato de las pacientes en el análisis, contándose con la aprobación de los comités de ética de las instituciones participantes. Se incorporaron únicamente las pacientes que dieron su consentimiento informado. En caso de que la paciente hubiese fallecido, se solicitó consentimiento para utilización de material de archivo (bloques de parafina) al familiar más cercano accesible.

\section{Resultados}

El análisis incluyó 40 de las 107 pacientes diagnosticadas de CM antes de los 40 años, en las que fue posible contar con la muestra tumoral parafinada para realizar técnicas IHQ y con el consentimiento informado.

Veintisiete pacientes $(67,5 \%)$ fueron asistidas en el CASMU; 12 pacientes (30\%) en el CHPR, y $1(2,5 \%)$ en el Servicio de Oncología del Hospital de Clínicas.

La edad mediana al momento del diagnóstico fue de 36 años (rango 29 a 39 años), la mayoría de las pacientes eran premenopáusicas al diagnóstico (22 pacientes, $55 \%), 13$ fueron perimenopáusicas $(32,5)$ y en el resto de las pacientes no se contaba con el dato. En relación con los factores de riesgo para el desarrollo de la enfermedad, considerando factores de riesgo hormonales: 5 pacientes $(12,5 \%)$ tuvieron menarca precoz (antes de los 12 años), 5 (12,5\%) fueron nuligestas y 8 pacientes (20\%) tuvieron AF significativos de CM u ovario. El ti- 
Tabla 1. Características clínico-patológicas, estadio, $\mathrm{RRHH}$ y HER 2 de las pacientes incluidas en el estudio $(n=40)$.

\begin{tabular}{|c|c|c|c|}
\hline Variables & & & $\%$ \\
\hline Edad mediana & 36 años & 40 & 100 \\
\hline \multicolumn{4}{|l|}{ Estatus menopáusico } \\
\hline Premenopáusicas & & 22 & 55 \\
\hline Perimenopáusicas & & 13 & 32,5 \\
\hline Sin datos & & 5 & 12,5 \\
\hline
\end{tabular}

Tipo histológico

Carcinoma ductal infiltrante (CDI)

$37 \quad 92,5$

Grado histológico final (GHF)

\begin{tabular}{lrr} 
GHF II & 12 & 30 \\
GHF III & 21 & 52,5 \\
\hline
\end{tabular}

Tamaño tumoral

\begin{tabular}{rrr} 
T1 & 13 & 32,5 \\
T2 & 20 & 50 \\
T3 & 6 & 15 \\
T4 & 1 & 2,5 \\
\hline
\end{tabular}

Estatus axilar

\begin{tabular}{rrr} 
N0 & 18 & 45 \\
N1 & 12 & 30 \\
N2 & 5 & 12,5 \\
N3 & 5 & 12,5 \\
\hline
\end{tabular}

Estadio

\begin{tabular}{lrr} 
I & 9 & 22,5 \\
II & 20 & 50 \\
III & 20 & 25 \\
IV & 10 & 2,5 \\
\hline
\end{tabular}

Estatus de RE y RP

\begin{tabular}{lcc}
$R E / R P+$ & 23 & 57,5 \\
RE- RP- & 17 & 42,5 \\
\hline
\end{tabular}

Estatus HER 2

\begin{tabular}{lcc} 
HER $2+(3+/ 2+$ FISH +$)$ & 13 & 32,5 \\
HER 2 - (1 + / 2 + FISH- $)$ & 27 & 67,5 \\
\hline
\end{tabular}

Tabla 2. Subtipos biológicos $(n=40)$.

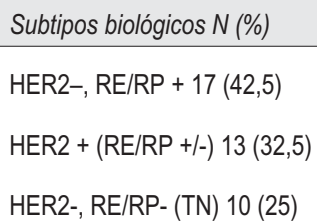

po histológico ductal infiltrante fue el más frecuente (37 pacientes, 92,5\%), con alto grado histológico (21 pacientes, 52,5\%). La mayoría de los tumores se encontraban limitados a la mama y la axila al momento del diagnóstico: el 82,5\% de las pacientes tuvieron tumores menores a $5 \mathrm{~cm}$ y el $75 \%$ no tuvo compromiso axilar o tuvo menos de tres ganglios metastásicos. El 72,5\% de las pacientes se presentaron en estadios precoces de la enfermedad. Las características clínico-patológicas de las pacientes se muestran en la tabla 1.

Con respecto al perfil biológico: 17 pacientes (42,5\%) fueron HER2-, RE/RP+, 13 (32,5\%) fueron HER $2+$ y las restantes 10 pacientes $(25 \%)$ fueron clasificadas como triple negativas (tabla 2).

En cuanto al tratamiento sistémico, 33 pacientes $(82,5 \%)$ recibieron quimioterapia y todas las mujeres con tumores mayores a $1 \mathrm{~cm}$ o axila positiva, HER 2 positivos, recibieron tratamiento con trastuzumab adyuvante. Todas las pacientes con CM RE o RP positivos, o ambos, recibieron hormonoterapia. Quince pacientes (37,5\%) presentaron recaída en la evolución, el 66,6\% (10 pacientes) de estas presentaron recaída sistémica exclusiva, $2(13,3 \%)$ presentaron recaída locorregional exclusiva y $3(20 \%)$ presentaron recaída locorregional y sistémica. En todas las pacientes que presentaron recaídas sistémicas se utilizó tratamiento sistémico en base a quimioterapia \pm terapias dirigidas $\mathrm{u}$ hormonoterapia. Las pacientes con recaídas locorregionales recibieron tratamiento local con cirugía \pm radioterapia y tratamiento sistémico en algún momento de su evolución.

La SVG a cinco años para el total de las pacientes fue de $73 \%$, mientras que la SVLE a cinco años fue de $60 \%$; no se alcanzó la mediana para la SVG, ni para la SVLE.

\section{Análisis de la expresión de BRCA1 por inmunohistoquímica}

La detección y localización de la proteína BRCA1 mediante IHQ se realizó utilizando dos anticuerpos monoclonales dirigidos contra diferentes regiones de la proteína, de acuerdo a lo descrito en Material y método (figura 1).

Dado que la pérdida de expresión de la proteína BRCA1 es uno de los mecanismos que puede estar en la 


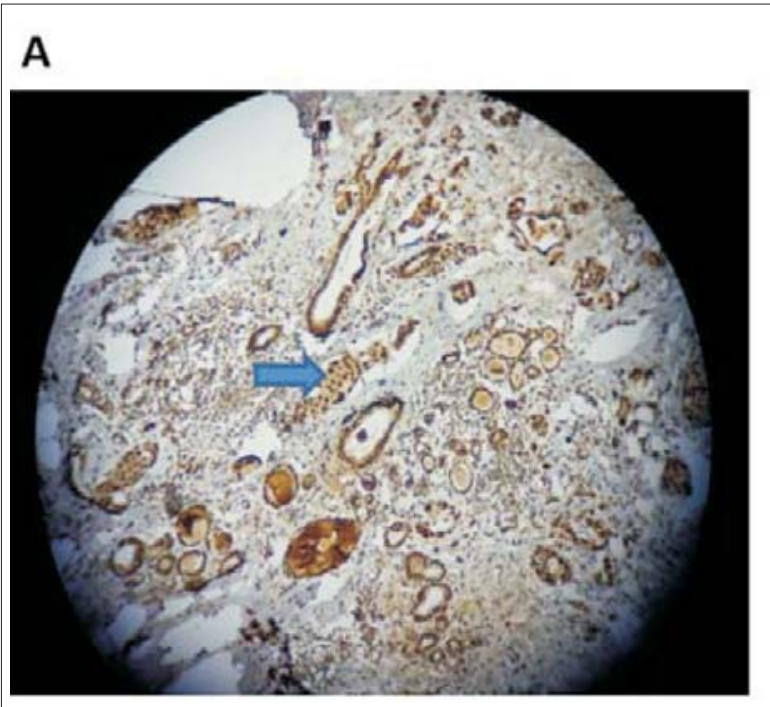

\section{B}

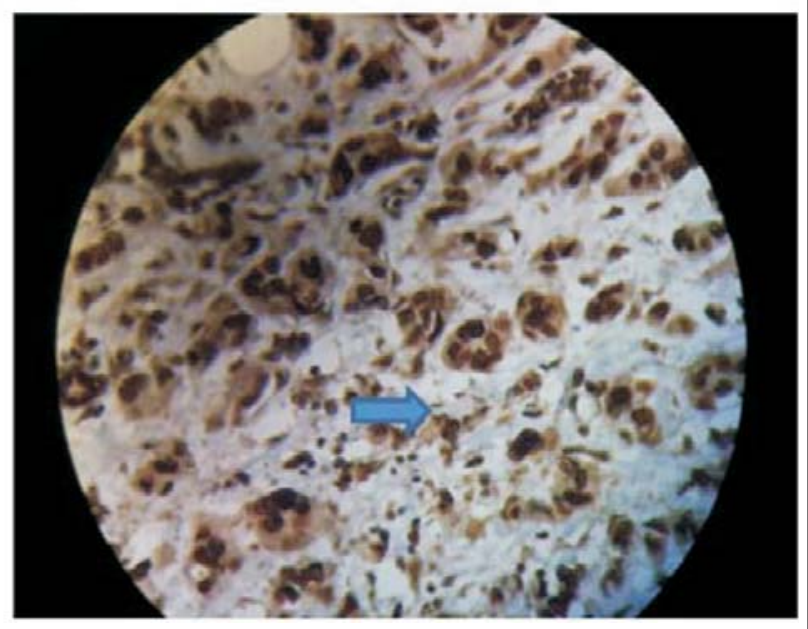

Figura 1. Técnica de IHQ: cortes de carcinomas mamarios incluidos en parafina en los cuales se observa la inmunomarcación a nivel nuclear y citoplasmático (flechas). A) Inmunomarcación utilizando el anticuerpo MS 110, dilución 1:100, 100 x. B) Inmunomarcación utilizando el anticuerpo GLK2, dilución 1:50, 400 x.

Tabla 3. Nivel de expresión de BRCA1.

\begin{tabular}{lcc}
\hline Expresión BRCA 1 & MS 110 & GLK2 \\
\hline$\leq 10 \%$ & $11(27,5 \%)$ & $16(40 \%)$ \\
$>10 \%$ & $29(72,5 \%)$ & $34(60 \%)$ \\
\hline
\end{tabular}

base de la carcinogénesis mamaria, nos centraremos en el análisis de los casos con una inmunomarcación $\leq 10 \%$ en los núcleos y el citoplasma de las células tumorales, considerados como negativos.

La expresión de BRCA1 fue negativa en 11 de las 40 pacientes incluidas $(27,5 \%)$ utilizando el anticuerpo MS1 10 y en 16 de las 40 pacientes incluidas (40\%) utilizando el anticuerpo GLK2 (tabla 3). En 8 casos (20\%) ambos anticuerpos presentaron una inmumomarcación $\leq 10 \%$ en los núcleos y el citoplasma de las células tumorales. Esto evidencia una discordancia en la inmunomarcación dado que esos casos representan el 72\% de los negativos para MS110, pero solo el 50\% de los negativos para GLK2.

\section{Expresión de BRCA1 y evolución de la enfermedad}

Durante el seguimiento, 14 pacientes presentaron una recaída de la enfermedad. Cuando se evaluó la expresión tumoral de BRCA1 en esas pacientes, se observó que la proporción de casos con inmunomarcación $\leq 10 \%$ fue mayor con el anticuerpo GLK2 (8/14, 57\%) que con el anticuerpo MS110 (5/14, 36\%), aunque la diferencia no fue estadísticamente significativa. Las cinco pacientes que presentaron inmunomarcación $\leq 10 \%$ con el anticuerpo MS 110 también presentaron inmumarcación $\leq 10 \%$ para GLK2.

Por otra parte, el porcentaje de recaídas fue mayor cuando ambas inmunomarcaciones fueron negativas $(5 / 8$, $62,5 \%$ ) que cuando lo fue solo para GLK2 (3/8, 37,5\%), o solo para MS110 (0/3), aunque la diferencia no fue estadísticamente significativa (chi cuadrado, $\mathrm{p}=0,39$ ).

Asimismo, se evaluó la sobrevida en función de la expresión de BRCA1 para cada anticuerpo. Para MS110 la SVG a cinco años para las pacientes con expresión tumoral $\leq 10 \%$ fue de $70 \%$ y de $79 \%$ para las pacientes con expresión $>10 \%$, no siendo esta diferencia estadísticamente significativa $(\mathrm{p}=0,122)$ (figura 2$)$.

La SVLE a cinco años para las pacientes con expresión tumoral $\leq 10 \%$ fue de $62 \%$ y de $55 \%$ para las pacientes con expresión $>10 \%$, no siendo esta diferencia tampoco estadísticamente significativa ( $p=0,297)$ (figura 3). Las medianas de SVLE y SVG se muestran en la tabla 4.

Para GLK2, la SVG a cinco años para las pacientes con expresión $\leq 10 \%$ fue de $56 \%$ y de $85 \%$ para las pacientes con expresión $>10 \%$, siendo esta diferencia estadísticamente significativa ( $\log$ rank, $p=0,015$ ) (figura 4). La SVLE a cinco años para las pacientes con expresión tumoral $\leq 10 \%$ fue de $40 \%$ y de $72 \%$ para las pacientes con expresión $>10 \%$, siendo esta diferencia también estadísticamente significativa (log rank, $\mathrm{p}=0,034$ ) (figura 5). Las medianas de SVLE y SVG se muestran en la tabla 4. 


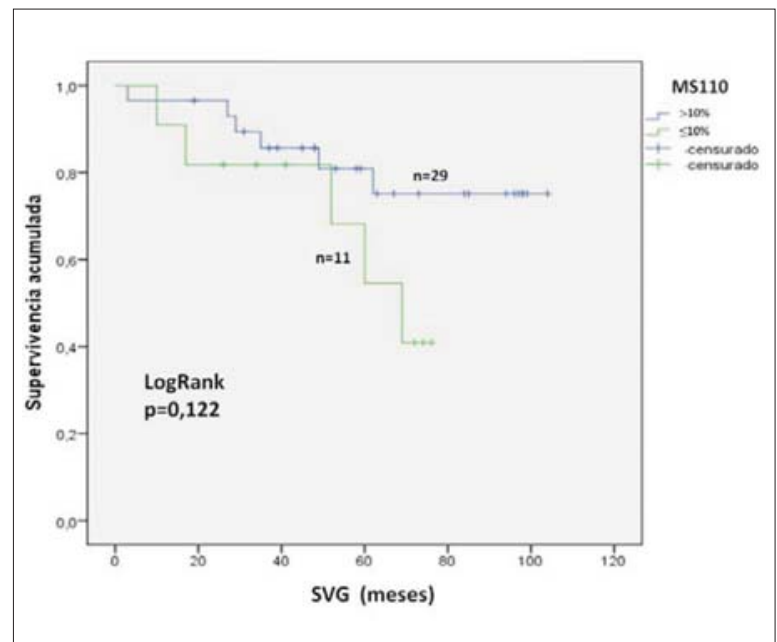

Figura 2. SVG a cinco años de acuerdo a la expresión tumoral de la proteína BRCA1 analizada mediante el anticuerpo MS110. La inmunomarcación se consideró positiva (>10\%, línea azul) o negativa ( $\leq 10 \%$, línea verde). La diferencia entre los grupos se consideró significativa con un valor de $\mathrm{p} \leq 0,05$ (test de log rank).

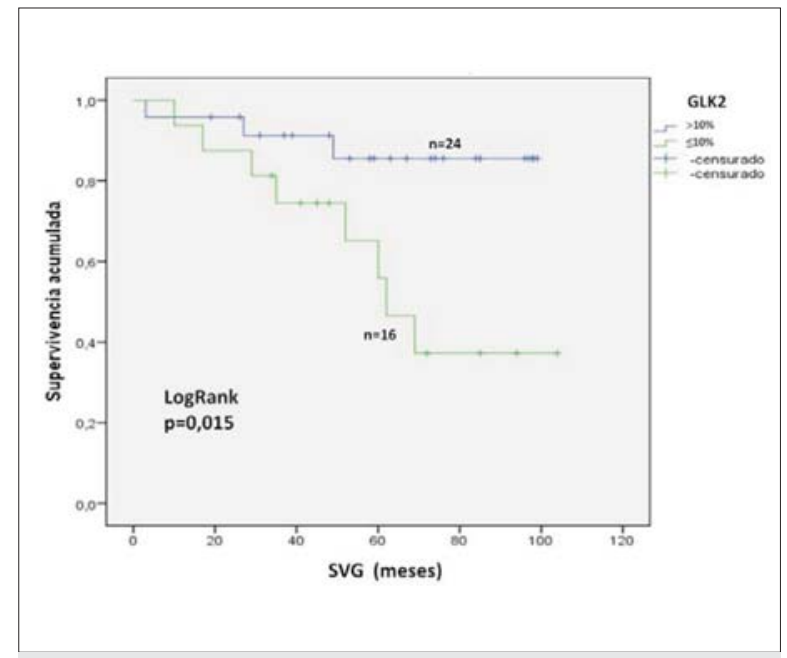

Figura 4. SVG a cinco años de acuerdo a la expresión tumoral de la proteína BRCA1 analizada mediante el anticuerpo GLK2. La inmunomarcación se consideró positiva ( $>10 \%$, línea azul) o negativa ( $\leq 10 \%$, línea verde). La diferencia entre los grupos se consideró significativa con un valor de $p \leq 0,05$ (test de log rank).

\section{Discusión}

Con respecto a las variables clínico-patológicas de las pacientes incluidas en este estudio, 20\% de las pacientes tenían AF significativos, lo cual, añadido a la temprana edad de aparición de cáncer en estas pacientes, hace pensar en una predisposición hereditaria al $\mathrm{CM}$ o

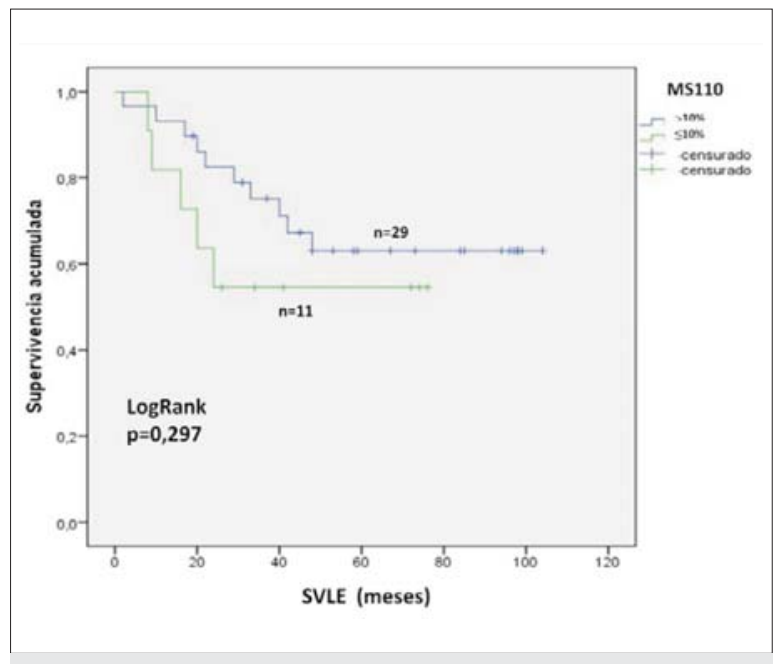

Figura 3. SVLE a cinco años de acuerdo a la expresión tumoral de la proteína BRCA1 analizada mediante el anticuerpo MS110. La inmunomarcación se consideró positiva (>10\%, línea azul) o negativa ( $\leq 10 \%$, línea verde). La diferencia entre los grupos se consideró significativa con un valor de $\mathrm{p} \leq 0,05$ (test de log rank).

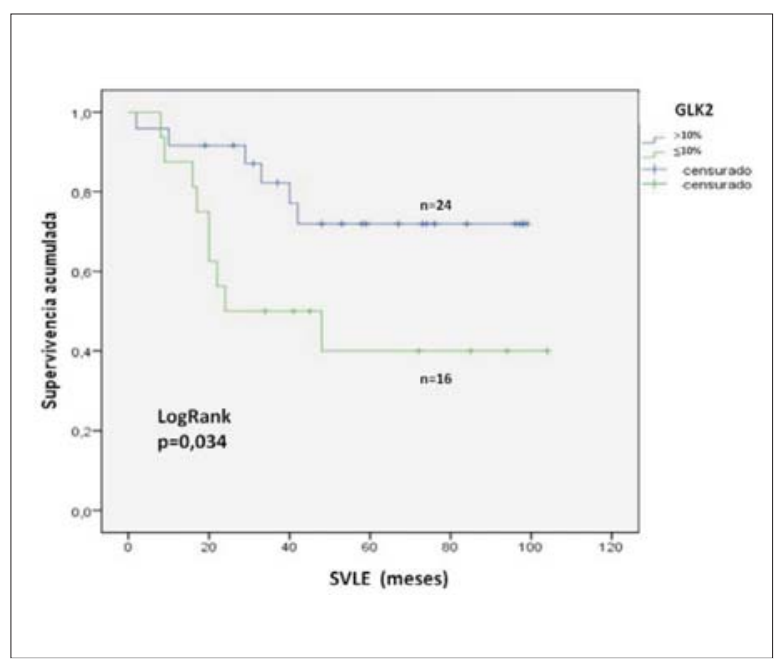

Figura 5. SVLE a cinco años de acuerdo a la expresión tumoral de la proteína BRCA1 analizada mediante el anticuerpo GLK2. La inmunomarcación se consideró positiva (>10\%, línea azul) o negativa ( $\leq 10 \%$, línea verde). La diferencia entre los grupos se consideró significativa con un valor de $p \leq 0,05$ (test de log rank)

de ovario, o ambos. Diversos trabajos muestran que las mutación en genes BRCA1 o 2 se presentan entre el $11,6 \%$ y el $17 \%$ de las mujeres con diagnóstico de $\mathrm{CM}$ menores de 40 años, siendo esta cifra similar a la hallada en nuestro estudio ${ }^{(12,13)}$. 
Tabla 4. Mediana de SVLE y SVG según nivel de expresión de MS110 y GLK2.

\begin{tabular}{ll}
\hline Mediana SVLE & Mediana SVG \\
\hline MS110 + no alcanzada & No alcanzada \\
MS110 - no alcanzada & 69 meses (IC 95\% 47-91) \\
GLK2 + no alcanzada & No alcanzada \\
GLK2 - 24 meses (IC 95\% 0-63) & 62 meses (IC 95\% 49-76) \\
\hline
\end{tabular}

Si confrontamos nuestros resultados con los reportados tanto en estudios nacionales ${ }^{(28)}$ como internacionales ${ }^{(29-30)}$ sobre frecuencia de subtipos biológicos para mujeres de todas las edades con $\mathrm{CM}$, nuestras pacientes presentaron mayor porcentaje de subgrupos biológicos agresivos HER $2+(32,5 \%)$ y TN $(25 \%)$. Estos resultados son coincidentes con lo evidenciado por Cancello y colaboradores en un estudio que incluyó mujeres menores de 35 años, donde los porcentajes de pacientes por subtipos biológicos fueron los siguientes: HER 2+ (21\%), TN $(16,2 \%)$ y receptores hormonales +/HER 2$(49,8 \%)^{(31)}$. La mayoría de nuestras pacientes presentaron tumores pequeños con estadios localizados I y II $(72,5 \%)$.

Como ya fue mencionado, aunque la mayor parte de los CM en la mujer joven son esporádicos, esta población tiene mayor riesgo de presentar una predisposición hereditaria para el desarrollo de la enfermedad, siendo los genes BRCA1 y 2 los más frecuentemente involucrados. En el Reino Unido se ha descrito, por ejemplo, que aproximadamente el $3 \%$ de todos los $\mathrm{CM}$ se atribuyen a mutaciones BRCA 1 o BRCA 2, siendo la proporción mayor en mujeres con diagnóstico antes de los 36 años, con un porcentaje que varía entre $6 \%$ y $9 \%{ }^{(22)}$.

A pesar de que se sabe que aproximadamente en 30\%-55\% de las pacientes portadoras de CM esporádico existe una pérdida de función del gen BRCA1 asociada a cambios epigenéticos ${ }^{(18,19)}$, no hemos encontrado estudios que reporten el nivel de expresión de BRCA1 en mujeres menores de 40 años con CM esporádico.

Dado que en las mujeres jóvenes no existe una clara caracterización molecular del CM, pensamos que la investigación en este sentido debería ser una prioridad de la investigación científica.

En lo que se refiere a la ubicación intracelular de la proteína BRCA1, su expresión exclusiva a nivel nuclear podría estar reflejando su fenotipo normal, ya que su sitio habitual es el núcleo celular, donde participa en la reparación del ADN, la transcripción, el control del ciclo celular y la apoptosis ${ }^{(20,21)}$. Si bien antes se pensaba que BRCA1 se encontraba a nivel nuclear y que la tinción ci- toplasmática era un artefacto técnico, en la actualidad se reconoce que debido a las mutaciones que pueden afectar al gen, la proteína BRCA1 permanece en el citoplasma celular y no se trasloca al núcleo celular, por lo que puede hallarse tanto a nivel nuclear como citoplasmáti$\mathrm{co}^{(32)}$.

En relación con la evolución de la enfermedad, 14 de las 40 pacientes incluidas en nuestro estudio (35\%) presentaron recaída durante el seguimiento. La mayoría de estas ( 8 pacientes, $57,1 \%$ ) presentaron inmunomarcación $\leq 10 \%$ con el anticuerpo GLK 2 y más de un tercio de las mismas ( 5 pacientes, $35,7 \%$ ) presentaron inmunomarcación $\leq 10 \%$ con el anticuerpo MS110. Es de destacar que las cinco pacientes con inmunomarcación MS110 negativa presentaron también inmunomarcación para GLK2 negativa. El hecho de que el porcentaje de recaídas fuera mayor cuando la inmunomarcación fuera negativa para ambos anticuerpos sugiere que la ausencia total de la proteína BRCA1, y, por lo tanto, de su función, se asocia con una mala evolución. En este sentido, la SVLE y la SVG fueron menores cuando la inmunomarcación fue negativa para GLK2. Sin embargo, los análisis de sobrevida de acuerdo a la inmunomarcación de MS110 no alcanzaron significación estadística, aunque la SVLE fue menor para las pacientes con inmunomarcación negativa. Este resultado podría ser explicado por el bajo número de pacientes, por lo que pensamos en un futuro poder incluir más pacientes en el estudio, para darle mayor poder estadístico.

Los resultados obtenidos con GLK2 coinciden con lo reportado por Kim y colaboradores, quienes reportaron un pronóstico desfavorable al disminuir la expresión de BRCA1 en el núcleo y citoplasma de las células tumorales de la mama ${ }^{(26)}$, lo que no hace pensar que el nivel de expresión de BRCA1 por IHQ del anticuerpo monoclonal GLK-2 podría ser útil como marcador predictivo de recaída. Así, en nuestro estudio, el nivel de expresión de BRCA1 determinado mediante IHQ con GLK2 constituyó un factor de mal pronóstico, ya que las pacientes con expresión nuclear y citoplasmática $\leq 10 \%$ tuvieron menor SVLE y SVG, siendo esto concordante con estudios internacionales, donde se evidencia que en el CM hereditario y esporádico la disminución de la expresión de BRCA1 a nivel nuclear y citoplasmático se ha asociado a un peor pronóstico y a una menor supervivencia $^{(33-36)}$. A nivel nacional, en una comunicación previa, Zanetti y colaboradores reportan que bajos niveles de expresión tumoral del transcripto (ARNm) de BRCA1 se correlacionan con una peor evolución en 60 pacientes portadoras de $\mathrm{CM}$ esporádico ${ }^{(37)}$.

Esto podría ser explicado a la metilación aberrante del promotor del gen, que ocasiona la pérdida de su expresión y por ende de su función dentro de la célula. 
En nuestro estudio, este mecanismo podría estar involucrado en los casos con inmunomarcación negativa de ambos anticuerpos, lo que podría ser analizado en futuros trabajos. En los casos con inmunomarcación negativa exclusivamente de GLK2, dado que el anticuerpo reconoce el extremo carboxi-terminal de la proteína, podría deberse a la presencia de una mutación de línea germinal del gen que determine una proteína truncada, lo que debería confirmarse mediante secuenciación del gen.

Como limitantes debemos mencionar que solo fue posible incluir 40 de las 107 pacientes diagnosticadas de CM antes de los 40 años, lo que podría introducir un sesgo en nuestro estudio. Esto se explica porque se incluyeron únicamente a las pacientes que contaban con muestra tumoral parafinada para realizar técnicas IHQ y con consentimiento informado.

En este sentido debemos tener en cuenta el pequeño tamaño muestral, por lo que desafortunadamente no fue posible indagadar la asociación entre las variables clínico-patológicas, subtipos moleculares y el marcador IHQ, quedando esto pendiente para el futuro.

Por otro lado, el estudio incluyó pacientes asistidas en tres instituciones de Montevideo, lo que puede generar un sesgo en los resultados al momento de interpretarlos como representativos de la población nacional.

De esta forma, queda pendiente para estudios a futuro la evaluación de las características de la enfermedad en un mayor número de pacientes, incluyendo pacientes del interior del país.

Teniendo en cuenta el hecho de que aún no se tienen estudios preliminares publicados en Uruguay respecto a la expresión de BRCA1 en nuestra población, se requieren otras investigaciones con series más grandes para llegar a conclusiones sobre su posible utilidad pronóstica o predictiva.

\section{Conclusión}

La pérdida de expresión de BRCA1 mediante GLK-2, que sugiere la presencia de una proteína truncada, se asoció a una menor SVG y SVLE de forma estadísticamente significativa. Nuestros resultados concuerdan con lo reportado en la literatura y evidencian que la disminución de la proteína BRCA1 determinada mediante IHQ con la clona GLK2 podría tener un valor pronóstico desfavorable para las pacientes jóvenes portadoras de CM.

\section{Agradecimientos}

Agradecemos a Ana Araújo, técnica en Anatomía Patológica, quien contribuyó en el procesamiento de las muestras para IHQ, y al Dr. Alejandro Noria, ayudante del Departamento de Métodos Cuantitativos de la Fa- cultada de Medicina (UDELAR), quien colaboró en la realización de los cálculos estadísticos.

\section{Abstract}

BRCA1 mutations are rare in sporadic breast cancer (CM), however their expression at the tumor level is diminished or absent in $30-50 \%$ of cases.

Objective: to assess the tumor expression of BRCA1 using immunohistochemistry (IHC) in Uruguayan women diagnosed with $\mathrm{BC}$ before the age of 40 years.

Material and methods: patients diagnosed with BC before the age of 40 between. The antibodies used were anti BRCA1 MS110 monoclonal antibodies against the $\mathrm{N}$-terminal end and GLK-2 against the C-terminal. Overall survival (OS) and disease free survival (DFS) were calculated; the curves were developed using the Kaplan-Meier method and the difference in survival was evaluated through the log rank test.

Results: the average age of the 40 patients included was 36 years. The 5 -year OS and DFS were $73 \%$ and $60 \%$ respectively. The expression of BRCA1 with GLK-2 was $\leq 10 \%$ in 16 of the 40 patients included (40\%). The 5 -year OS and DFS for patients with $\leq 10 \%$ expression was $56 \%$ vs. $85 \%$ for patients with $>10 \%$ $(p=0.015)$ and $40 \%$ vs. $72 \%(p=0.034)$ respectively. The expression of BRCA 1 by MS1 10 was $\leq 10 \%$ in 11 of the 40 patients included (27.5\%). No differences were found in the 5-year OS or DFS based on the expression of this marker.

Conclusion: The loss of BRCA1 expression using GLK-2, which suggests the presence of a truncated protein, was associated with a statistically significantly lower OS and DFS, that the decrease in the BRCA1 protein as determined by GLK2 has an unfavorable prognostic value for young patients with $\mathrm{BC}$.

\section{Resumo}

As mutações de BRCA1 são raras no câncer de mama (CM) esporádico; no entanto sua expressão no nível tumoral está diminuída ou ausente em $30-50 \%$ dos casos.

Objetivo: avaliar a expressão tumoral de BRCA1 por imuno-histoquímica (IHQ) em mulheres uruguaias com diagnóstico de $\mathrm{CM}$ antes dos 40 anos.

Material e métodos: foram incluídas pacientes com diagnóstico de $\mathrm{CM}$ antes dos 40 anos. Foram utilizados anticorpos monoclonais anti BRCA1 MS110 contra o extremo N-terminal e GLK-2 contra o extremo C-terminal. A sobrevida global (SVG) e a sobrevida livre de enfermidade (SVLE) foram calculadas; o método de Kaplan-Meier foi utilizado para a construção das curvas e a diferença de sobrevida foi avaliada usando o teste de log-rank. 
Resultados: foram incluídas 40 pacientes; a SVG e a SVLE aos 5 anos foram $73 \%$ e $60 \%$ respectivamente. A expressão de BRCA1 mediante GLK-2 foi $£ 10 \%$ em 16 das 40 pacientes ( $40 \%$ ). A SVG e a SVLE aos 5 anos para as pacientes com expressão $\leq 10 \%$ foi $56 \%$ vs. $85 \%$ para as pacientes com expressão $>10 \%(\mathrm{p}=0,015)$ e $40 \%$ vs. $72 \%(\mathrm{p}=0,034)$ respectivamente. A expressão de BRCA1 mediante MS110 foi $\leq 10 \%$ em 11 das 40 pacientes $(27,5 \%)$. Não foram encontradas diferenças na SVG nem na SVLE aos 5 anos com este marcador.

Conclusão: foi encontrada perda da expressão tumoral de BRCA1 determinada por GLK-2 em 40\% das pacientes incluídas e foi associada a uma menor SVG e SVLE, o que poderia ter um valor prognóstico desfavorável nestas pacientes.

\section{Bibliografía}

1. Barrios E, Garau M. Cáncer: magnitud del problema en el mundo y en Uruguay, aspectos epidemiológicos. An Facultad Med (Univ Repúb Urug) 2017; 4(1):9-47.

2. Barrios E, Garau M, Alonso R, Musetti C. IV Atlas de incidencia del cáncer en el Uruguay 2007-2011. Montevideo: Comisión Honoraria de Lucha Contra el Cáncer, 2014.

3. Adami H, Malker B, Holmberg L, Persson I, Stone B. The relation between survival and age at diagnosis in breast cancer. N Engl J Med 1986; 315(9):559-63.

4. Kroman N, Jensen M, Wohlfahrt J, Mouridsen H, Andersen P, Melbye M. Factors influencing the effect of age on prognosis in breast cancer: population based study. BMJ 2000; 320(7233):474-8.

5. Ahn S, Son B, Kim S, Kim S, Jeong J, Ko S, et al. Poor outcome of hormone receptor-positive breast cancer at very young age is due to tamoxifen resistance: nationwide survival data in Korea- a report from the Korean Breast Cancer Society. J Clin Oncol 2007; 25(17):2360-8.

6. Francis P. Optimal adjuvant therapy for very young breast cancer patients. Breast 2011; 20(4):297-302. doi:10.1016/j.breast.2011.05.002

7. Anders C, Hsu D, Broadwater G, Acharya C, Foekens J, Zhang Y, et al. Young age at diagnosis correlates with worse prognosis and defines a subset of breast cancers with shared patterns of gene expression. J Clin Oncol 2008; 26(20):3324-30. doi: 10.1200/JCO.2007.14.2471

8. Anders C, Fan C, Parker J, Carey L, Blackwell K, Klauber-DeMore $\mathbf{N}$, et al. Breast carcinomas arising at a young age: unique biology or a surrogate for aggressive intrinsic subtypes? J Clin Oncol 2011; 29(1):e18-20. doi: 10.1200/JCO.2010.28.9199

9. Narod S. Breast cancer in young women. Nat Rev Clin Oncol 2012; 9(8):460-70.

10. Cardoso F, Loibl S, Pagani O, Graziottin A, Panizza P, Martincich L, et al. The European Society of Breast Cancer Specialists recommendations for the management of young women with breast cancer. Eur J Cancer 2012; 48(18):3355-77. doi: 10.1016/j.ejca.2012.10.004

11. Malvasio S, Schiavone A, Camejo N, Castillo C, Artgaveytia N, Di Matteo C, et al. Características clínico-patológicas y evolución del cáncer de mama en mujeres uruguayas jóvenes . Rev Méd Urug 2017; 33(2):94-101.

12. de Sanjosé $\mathbf{S}$, Léoné $M$, Bérez $\mathbf{V}$, Izquierdo A, Font R, Brunet J, et al. Prevalence of BRCA1 and BRCA2 germline mutations in young breast cancer patients: a population-based study. Int J Cancer 2003; 106(4):588-93.

13. Newman B, Mu H, Butler L, Millikan R, Moorman P, King M. Frequency of breast cancer attributable to BRCA1 in a population-based series of American women. JAMA 1998; 279(12):915-21.

14. Frank T, Deffenbaugh A, Reid J, Hulick M, Ward B, Lingenfelter B, et al. Clinical characteristics of individuals with germline mutations in BRCA1 and BRCA2: analysis of 10,000 individuals. J Clin Oncol 2002; 20(6):1480-90.

15. Delgado L, González A, Fernández G, Cataldi S, Bressac-de Paillerets B, Bombled J, et al. BRCA1 and BRCA2 mutations in Uruguayan breast and breast/ovarian cancer families. Proc Am Soc Clin Oncol 2003; 22: abstr 3510.

16. Fodor F, Weston A, Bleiweiss I, McCurdy L, Walsh M, Tartter P, et al. Frequency and carrier risk associated with common BRCA1 and BRCA2 mutations in Ashkenazi Jewish breast cancer patients. Am J Hum Genet 1998; 63(1):45-51.

17. Thorlacius S, Struewing J, Hartge P, Olafsdottir G, Sigvaldason $\mathbf{H}$, Tryggvadottir $\mathbf{L}$, et al. Population-based study of risk of breast cancer in carriers of BRCA2 mutation. Lancet 1998; 352(9137):1337-9.

18. Kurebayashi J, Yamamoto Y, Kurosumi M, Okubo S, Nomura T, Tanaka K, et al. Loss of BRCA1 expression may predict shorter time-to-progression in metastatic breast cancer patients treated with taxanes. Anticancer Res 2006; 26(1B):695-701.

19. Roldán G, Delgado L, Musé I. Tumoral expression of BRCA1, estrogen receptor alpha and ID4 protein in patients with sporadic breast cancer. Cancer Biol Ther 2006; 5(5):505-10.

20. Narod S, Foulkes W. BRCA1 and BRCA2: 1994 and beyond. Nat Rev Cancer 2004; 4(9):665-76.

21. Yoshida K, Miki Y. Role of BRCA1 and BRCA2 as regulators of DNA repair, transcription, and cell cycle in response to DNA damage. Cancer Sci 2004; 95(11):866-71.

22. Peto J, Collins N, Barfoot R, Seal S, Warren W, Rahman $\mathbf{N}$, et al. Prevalence of BRCA1 and BRCA2 gene mutations in patients with early-onset breast cancer. J Natl Cancer Inst 1999; 91(11):943-9.

23. Zhang Q, Zhang $\mathbf{Q}$, Cong $\mathbf{H}$, Zhang $X$. The ectopic expression of BRCA1 is associated with genesis, progression, and prognosis of breast cancer in young patients. Diagn Pathol 2012; 7:181.

24. Shimizu Y, Luk H, Horio D, Miron P, Griswold M, Iglehart D, et al. BRCA1-IRIS overexpression promotes for- 
mation of aggressive breast cancers. PLoS One 2012; 7(4):e34102. doi: 10.1371/journal.pone.0034102

25. Madjd Z, Karimi A, Molanae S, Asadi-Lari M. BRCA1 Protein Expression Level and CD44(+)Phenotype in Breast Cancer Patients. Cell J 2011; 13(3):155-62.

26. Kim D, Jung W, Koo J. The expression of ERCC1, RRM1, and BRCA1 in breast cancer according to the immunohistochemical phenotypes. J Korean Med Sci 2011; 26(3):352-9.

27. Ansquer Y, Mandelbrot L, Lehy T, Salomon L, Dhainaut C, Madelenat P, et al. Expression of BRCA1, HER-1 (EGFR) and HER-2 in sporadic breast cancer and relationships to other clinicopathological prognostic features. Anticancer Res 2005; 25(6C):4535-41.

28. Delgado L, Fresco R, Santander G, Aguiar S, Camejo N, Ferrero L, et al. Expresión tumoral de HER-2, receptores de estrógenos y de progesterona y su relación con características clínico-patológicas en pacientes uruguayas con cáncer de mama. Rev Méd Urug 2010; 26(3):145-53.

29. Blows F, Driver K, Schmidt M, Broeks A, van Leeuwen F, Wesseling J, et al. Subtyping of breast cancer by immunohistochemistry to investigate a relationship between subtype and short and long term survival: a collaborative analysis of data for 10,159 cases from 12 studies. PLoS Med 2010; 7(5):e1000279. doi: 10.1371/journal.pmed.1000279

30. Gutierrez C, Schiff R. HER2: biology, detection, and clinical implications. Arch Pathol Lab Med 2011; 135(1):55-62.

31. Cancello G, Maisonneuve P, Rotmensz N, Viale G, Mastropasqua M, Pruneri G, et al. Prognosis and adjuvant treatment effects in selected breast cancer subtypes of very young women ( $<35$ years) with operable breast cancer. Ann Oncol 2010; 21(10):1974-81.

32. Wilson C, Ramos L, Villaseñor M, Anders K, Press M, Clarke K, et al. Localization of human BRCA1 and its loss in high-grade, non-inherited breast carcinomas. Nat Genet 1999; 21(2):236-40

33. Li Q, Wei W, Jiang Y, Yang H, Liu J. Promoter methylation and expression changes of BRCA1 in cancerous tissues of patients with sporadic breast cancer. Oncol Lett 2015; 9(4):1807-13

34. Yamashita N, Tokunaga E, Kitao H, Hitchins M, Inoue $\mathbf{Y}$, Tanaka K, et al. Epigenetic inactivation of BRCA1 through promoter hypermethylation and its clinical importance in triple-negative breast cancer. Clin Breast Cancer 2015; 15(6):498-504..

35. Hsu N, Huang Y, Yokoyama K, Chu P, Chen F, Hou M. Methylation of BRCA1 promoter region is associated with unfavorable prognosis in women with early-stage breast cancer. PLoS One 2013; 8(2):e56256. doi: 10.1371/journal.pone. 0056256

36. Pinto R, De Summa S, Pilato B, Tommasi S. DNA methylation and miRNAs regulation in hereditary breast cancer: epigenetic changes, players in transcriptional and post- transcriptional regulation in hereditary breast cancer. Curr Mol Med 2014; 14(1):45-57.

37. Zanetti J, Manrique G, Heinzen S, Cancela P, Alonso I, Artagaveytia N. Nivel de ARNm-BRCA1 como factor pronóstico en cáncer de mama esporádico. En: $63^{\mathrm{a}}$ Semana Académica del Hospital Universitario Dr. Manuel Quintela. Montevideo, 19-23 de septiembre de 2016.

\section{Contribución de autores}

Silvina Malvasio, https://orcid.org/0000-0003-0718-3113. Concepción y diseño, ejecución, análisis de datos e interpretación de resultados, redacción, revisión crítica, aprobación de la versión final.

Natalia Camejo, https://orcid.org/0000-0002-8684-0291. Concepción y diseño, ejecución, análisis de datos e interpretación de resultados, redacción, revisión crítica, aprobación de la versión final.

Carina Di Matteo, https://orcid.org/0000-0001-7210-6611. Ejecución, análisis de datos e interpretación de resultados, aprobación de la versión final.

Benedicta Caserta, https://orcid.org/0000-0001-8446-3616. Ejecución, análisis de datos e interpretación de resultados, aprobación de la versión final.

Nora Artagaveytia, https://orcid.org/0000-0001-8798-7941. Concepción y diseño, análisis de datos e interpretación de resultados, redacción, aprobación de la versión final, revisión crítica.

Cecilia Castillo, https://orcid.org/0000-0002-0417-0512. Concepción y diseño, aprobación de la versión final.

Andrea Schiavone, https://orcid.org/0000-0001-9018-6264. Ejecución, aprobación de la versión final.

Roberto Notejane, https://orcid.org/0000-0003-0063-355. Ejecución, aprobación de la versión final.

Isabel Alonso, https://orcid.org/0000-0001-5195-358X. Ejecución, aprobación de la versión final.

Lucía Delgado, https://orcid.org/0000-0002-2966-5490. Concepción y diseño, análisis de datos e interpretación de resultados, revisión crítica, aprobación de la versión final. 